UNIFICATION THROUGH A SUPERGROUP

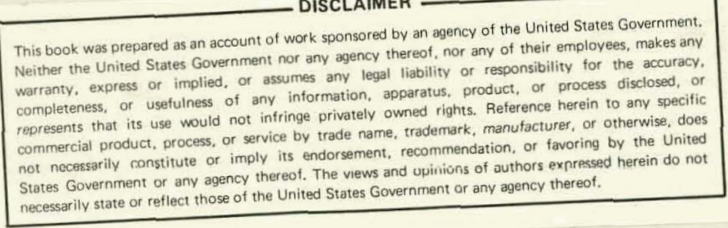

\section{MOTIGE Th}

\section{POATHOHS OF THIS REPOAT ARE ILLEERL.}

\section{it has been reproduced frem the best available copy to permit the broadest possible avaliability.}

\author{
Yuval Ne'eman $+*+"$ \\ Department of Physics and Astronomy \\ Tel Aviv University, Tel Aviv, Israel
}

\begin{abstract}
The representations of supergroups provide the most precise fit to the known set of fundamental physical matter fields. SU $(2 / 1)$ describes the unified weak-electromagnetic interactions and $\mathrm{SU}(5+k / 1)$ contains SU(3)color and $2^{k}$ exact "generations". Moreover, SU(2/1) predicts that the 4 th states of the lepton multiplets (i.e. $\nu_{R}^{0}$ ) de couple, whereas they don't in quark multiplets and $S U(n / l)$ predicts that the color degree of freedom is an SU(3). We provide all relevant constructions.
\end{abstract}

$\uparrow$ also Center for Particle Theory, University of Texas Austin, Texas, U S A.

* Wolfson Chair Extraordinary in Theoretical Physics

+ supported in part by the U.S. - Israel Binational Science Foundation

" supported in part by the U.S. D.O.E. contract EY-76-S-05-3992 


\section{DISCLAIMER}

This report was prepared as an account of work sponsored by an agency of the United States Government. Neither the United States Government nor any agency Thereof, nor any of their employees, makes any warranty, express or implied, or assumes any legal liability or responsibility for the accuracy, completeness, or usefulness of any information, apparatus, product, or process disclosed, or represents that its use would not infringe privately owned rights. Reference herein to any specific commercial product, process, or service by trade name, trademark, manufacturer, or otherwise does not necessarily constitute or imply its endorsement, recommendation, or favoring by the United States Government or any agency thereof. The views and opinions of authors expressed herein do not necessarily state or reflect those of the United States Government or any agency thereof. 


\section{DISCLAIMER}

Portions of this document may be illegible in electronic image products. Images are produced from the best available original document. 
1. SU(2/1) and the quantum numbers of trie spinor fieldis

It has been suggested 1) 2) that the supergroup $\mathrm{SU}(2 / 1)$, when gauged in a way appropriate to an internal supergauge, generates the unified electroweak (or asthenodynamic) interaction. The resulting Lagrangian roughly corresponds to a highly restricted Salam-Weinberg SU(2) $\left.{ }_{L} X\right) U(1)$ model.

We have applied a theorem stating that the assignment of different gracings 3 ), within the spinor field vector space $v$, between $v_{L}$ (left) ana $v_{R}$ ( $r i g h t$ ) chiralities, ensures that all matrices of su(2) $\otimes u(1)$ be supertraceless.

$\operatorname{str} M=\operatorname{tr} M_{L}-\operatorname{tr} M_{R}=0$

Proof: (a) The electric charges of any components are reflected

$Q\left(v_{L}^{m}\right)=Q\left(v_{R}^{m}\right)$

$\therefore \operatorname{str} M\{Q\}=0$

(b) $\operatorname{tr} M_{L}\left\{s u(2) L=0, M_{R}\left\{\operatorname{su}(2){ }_{L}\right\} \equiv 0\right.$

(c) $Q=I_{3}^{L}+L_{U}$

( $I_{3}^{L}$ is the third component of the weak left-handed isospin, $U$ is the weak hypercharge). Thus, since (b) implies $\operatorname{str} M\left\{I_{3}\right\}=0$,
we have

$$
\operatorname{str} M\{U\}=0
$$

Postulating that supertracelessness rerlects the presence of a supergroup. we have assumed that this is $\mathrm{SU}(2 / 1)$. The defining (irredyfible) representation $D\left(\frac{1}{2}, \frac{1}{2}\right)$ in the notation of scheunert et al (which we denote as SNR in this paper) carries the eigenvalues

$$
\begin{aligned}
& I_{3}^{L}=\operatorname{diag}(h,-h / 0), U=\operatorname{diag}(1,1 / 2) \\
& \text { fitting the antileptons }\left(\overline{\left(\bar{e}_{L}^{-}\right)_{R^{\prime}}^{+}}, \overline{\left(v_{L}^{0}\right)_{R}} / \overline{\left(\bar{e}_{R}^{-}\right)_{L}^{+}}\right)
\end{aligned}
$$

Note that in ref. ${ }^{1)}$ we had picked the lepton eigenvalues for our definitions. Our present choice (1.3) provides for positive eigenvalues. It has the adiitional advantage of fitting the SNR notation $D\left(\frac{1}{m} \alpha_{1} i \frac{L}{3}\right.$ max $)$ characterizing the eigenvalues of the state with highest $U$, within the set with highest If. The $\operatorname{SU}(2 / 1)$ hypothesis scores highly in providing us with a (star-hermitean) irreducible representation. $D(1 / 6,1 / 2)$ fitting the quarks

$$
\left(u_{L}^{2 / 3}, d_{L}^{1 / 3} / d_{R}^{-1 / 3}, u_{R}^{2 / 3}\right) \text { precisely }{ }^{5)},
$$




$$
\left.\begin{array}{l}
D\left(1 / 6,1 / 2 ; I_{3}^{L}\right)=\operatorname{diag}(1 / 2,-1 / 2 / 0,0) \\
D(1 / 6,1 / 2 ; U)=\operatorname{diag}(1 / 3,1 / 3 /-2 / 3,4 / 3)
\end{array}\right\}
$$

Note that the finite representations of $S u(2 / 1)$ are those of SL $(2 / 1)$, and had been listed 4$)$ by SNR prior to the formulation of our theory. The definition of unitarity for $S U(2 / 1)$ is based on (id. is the identity matrix)

$$
M^{+}=\left(M^{T}\right)^{X}, M^{+} M=i d \text {. }
$$

where $\mathrm{M}^{\mathrm{T}}$ is orainary transposition, and $\mathrm{x}$ denotes complex conjugation with inversion of the order of Grassmann generatingelements within each matrix-element. As long as we do not have a complete quantum theory of internal supergauges, we do not know how relevant Unitarity and hermiticity are in our representations. condition (1.1) ensures super unimodularity

$$
\operatorname{sdet} M:=\operatorname{det}\left(M_{R}\right) \cdot \operatorname{det}\left(M^{-1}\right)_{L}=1
$$

Note that the 4-dimensional representation in (1.4) depends on a parameter 6) fixing the $t_{\text {qu }}=1 / 6$ SNR eigenvalue. This parameter $b$ translates the eigenvalues of $D(U)$, without affecting the dimension. Taking, for reasons that we shall touch upon later, $b \stackrel{=}{=}$ for the leptons (and more generaily, bu mid $=b-\frac{1}{2}$ )

$$
\begin{aligned}
& \left.\begin{array}{l}
D\left(-\frac{1}{2}, I_{3} ; I_{3}^{L}\right)=\operatorname{diag}\left(\frac{1}{2},-\frac{1}{2} / 0,0\right) \\
D\left(-\frac{1}{2}, \frac{1}{2} ; U\right)=\operatorname{diag}(-1,-1 /-2,0)
\end{array}\right\} b=0 \\
& \text { as befits }\left(\nu_{L}^{0}, e_{L}^{-} / e_{R^{\prime}}^{-} \nu_{R}^{0}\right) \\
& \text { we get for } b=1 \text {, the } 4 \text { antileptons }\left(\overline{\left(\bar{e}_{L}^{-}\right)_{R}^{+}}, \overline{\left(\bar{\nu}_{L}^{0}\right)_{R}},\left(\overline{\left(\nu_{R}^{0}\right)_{L}}, \overline{\left(e_{R}^{-}\right)_{L}}\right)\right.
\end{aligned}
$$
given by $D\left(\frac{1}{2}, \frac{1}{2}\right)$.

The quarke (1.4) corrcspond to $6.2 / 3$, and the antiquarks

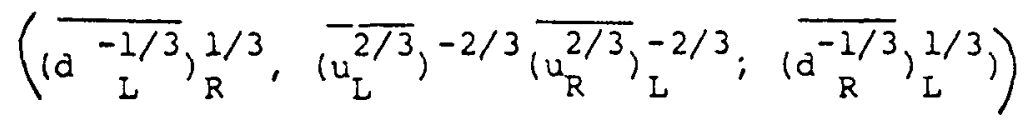

to $b=1 / 3$ or $D(-1 / 6,1 / 2)$.

Note that the representations in which the absolute values of the two SNR quantum numbers coincide, or alternatively in which $b$ is an integer, are reducible. They are not completely reducible, but $b=1$ has the 3-dimensional set (1.3) as an invariant subspace. Integer $b$ implies integer $U$ (and even $U$ for $v_{p}$ ), i.e. integer electric charges $Q$, as fixed by $(1.2)$. SU( 271$)$ may thus be said to predict that for integer charges, one state 
$\left(v_{R}^{\circ}\right.$ or $\left(\overline{\left(v_{R}^{0}\right)}\right)$ disconnects.

\section{Physical interpretation of the vector-space statistics}

our introduction of $\mathrm{SU}(2 / 1)$ was motivated by the finding 7) that the ghosts $X a$, introduced in the quantization procedure of a Yang- Mills theory, correspond to proper geometric "classical" objects, natural to the Principal Fiber Bundle description 8). we refer the reader to the rederivation of the Becchi-Rouet-Stora equations 9),

$$
\begin{aligned}
& \left(w_{\mu}^{a} \text { is the Yang-Mills field potential, } \psi^{m} \text { a matter field }\right) \\
& s w_{\mu}^{a}=\left(D_{\mu} X\right)^{a} \\
& s X^{a}=-\frac{2}{y}[X, X]^{a} \\
& s v^{m}=[X, v]^{m}=: \psi^{m} \\
& s^{2} w_{\mu}^{a}=0, s^{2} X^{a}=0, s^{2} v^{m}=0
\end{aligned}
$$

in which the ghosts $X^{\bar{a}}$ are seen to represent the "vertical" pieces of the complete connection on the principal bundle $P(M, G)$. We use equations (2.3) to define an "effective". matter-ghost $\psi^{\mathrm{m}}$, with statistics opposed to those of $\mathrm{v}^{\mathrm{m}}$. Note that in the original derivation (10),

$$
s=\frac{-\partial}{\partial \lambda} \delta_{B}
$$

in its action on $w_{\mu}^{a}, x^{a}$ and $\psi^{m}$. Here $\delta_{B}$ represents an

oroinary gauge transformation, in which the parameter $\alpha^{2}(x)$

has been factorized into two fermionic factors,

$$
\alpha^{a}(x)=x^{a}(x) \lambda
$$

where $\lambda$ is a constant Grassmann anticommuting element. In our classical treatment 1$), 5)$ we extended equations $(2.1-2.4)$ to the case of a supergroup (with a detailed presentation in rei.11). All multiplets take on new components with opposite grading (assuming that $G$ in $(2.1-2.4)$ is the maximal even subgroup of the supergroup of ). We now write 
$\left(\begin{array}{c}w_{\mu}^{a} \\ x_{\mu}^{2}\end{array}\right)=\varepsilon_{\mu}\left(\begin{array}{l}x^{a} \\ w^{i}\end{array}\right)$

$s\left(\begin{array}{c}v^{m} \\ \psi^{r}\end{array}\right)=\left(\begin{array}{c}\psi^{m} \\ v^{r}\end{array}\right)$

where $\varepsilon_{\mu}=s^{-1} D$. . a formal solution of (2.1).

$x_{\mu}^{i}$ is a new vector-gnost (fermionic, like $D_{\mu} X^{i}$ in (2.1)

and $w^{i}$ is the Golastone-Higgs multiplet. In su(2/1) it

has the correct phenomenological assignment!

We use latin letters for physical fields and greek for ghosts. The indices $a, i$ and $m, x$ represent even-odd components in the algebra and in the matter representations respectively. The action of $s$ may be inverted, as can be seen in (2.5a). Equation (2.7) and its inverse allow us to interpret the representations of $s \&(2 / 1)$. Each of $b=0,1$ and $b=2 / 3,1 / 3$ have to appear twice, with inverted statistics, as on both sioes of (2.7). In the first, the field $v^{m}$ corresponding to the hignest weight is a physical chiral spinor and the components with opposite gradings are ghost-fields $\psi^{T}$ related by $s$ to the physical $v$ in the second representation. Similarly, $s$ acting on $v^{m}$ generates the ghosts $\psi^{m}$ which fill out the highest weight chirality in that second representation. This is known as our "ghost interpretation" of the supergroup and its representation. We shall not deal here with the gauge-fields $(2.6)$, but note that our classical treatment 1$), 5$ y yielded $\frac{4}{3} g^{2}$ for the $w^{4}$ coupling, and $250 \mathrm{GeV}$ for the Higgs field's mass. We also had $\sin ^{2} \theta_{w}=\frac{1}{\text { as }}$ the unrenormalized Weinoerg angle, with $a$. conjecture that it is not renormalized except for the $\mathrm{SU}(2 / 1)$ breakdown itself (i.e. $2100 \mathrm{GeV}$ rather than $1015 \mathrm{GeV}$ of GuTs).

Without a quantum treatment, these results should be considered as tentative. We encountered some difficulties in dealing with the gauge-multiplet. The Killing metric

$$
g_{A B}=\operatorname{str}\left(M_{A} M_{B}\right)
$$

is not positive-definite in the guu even direction (that of the $U(1)$ in the even subgroup $\left.\left.S U(2)_{L} X\right) U(1)\right)_{4}$ It is also antisymmetric in the odd directions (whereas $w^{4}$ requises a symmetric metric). We deal with these issues elsewhere and prefer to concentrate on the kinematical aspects, which are as spectacular in $s u(2 / 1)$ as they were in the original unitary symetry hadron su( 3 ). 
However, our gnost interpretation is not tine oniy one which has been suggested. Other interpretations nave been proposed, based on extending space-time by acaitionai Bose 2) 12) or Fermi dimensions 13,14,15), etc. The results presented in the rest of this paper are independent of the ghost conjecture and can be adopted in either interpretation.

3. The fundamental representations of $\mathrm{s} \ell(\mathrm{n} / \mathrm{m})$

In a recent study 6$)$, we have constructed a class of "fundamental" representations of $s h(\mathrm{n} / \mathrm{m})$. These resemble the representations we used 17) to describe the supergravity gauge multiplet. For $m \neq l$, they are given by ( $k$ as an integer)

$$
\Lambda^{0}(v) \otimes S^{k}(x) \oplus \Lambda^{1}(v) \otimes S^{k-1} \oplus \ldots \oplus \Lambda^{n}(v) \otimes S^{k-n}(x)
$$

where $\Lambda(V)$ is the Grassmann manifold constructed on the fermi subspace $V=\wedge$ of the defining vector-space $w=V+X$. The $s^{k-i}$ is the space of homogeneous polynomials of degree $k-i$ on $x^{*}$ and $s^{j}=0$ for $j \times 0$. In the special case $m \doteq \operatorname{dim} x=1,(3.1)$ can be replaced by

$$
\Lambda^{0}(v) \otimes F^{b} \oplus \Lambda^{1}(V) \otimes F^{b-1} \oplus \cdots \oplus \Lambda^{n}(V) \otimes F^{b-n}
$$

where $b \in \mathbb{C}$ and $F^{b-i}$ is the one dimensional space of possibly multivalued functions defined on $x$ and homogeneous of degree $b-i$. The dimensionality of $(3.2)$ is. $2^{n}$, the same as tinat of $\wedge(V)$. For (3.1) we recover the defining representation by taking $k=1$ since we are left with

\section{$\Lambda^{\circ}(V) \operatorname{Qx} \oplus \Lambda^{1}(V) \otimes \mathbb{C}$}

In extended Supergravity the odd generators behave like $\underline{\mathrm{n}}$ of So $(n)$. and have helicity $j_{z}=$. One starts with the up-graviton with $j_{z}=2$ and acts with the odd annihilation operators once, getting $n \quad j_{z}=3 / 2$ states behaving like $\underline{n}$ etc.

Here, working with the simpler $S L(n) \supset S U(2 / 1)$, we have altogether 3 parameters : 2 in picking the eigenvalues of $U$ in the defining representation, and the quantum number $b$. We are given the highest weight, i.e. $u_{\mathrm{mid}}$. Assuming for $s \&(n / 1)$

$$
I_{3}^{L}=\operatorname{diag}(\frac{1}{2},-\frac{1}{2}, \underbrace{0, \ldots 00)}_{n-2 \text { zeros }}
$$

and 


$$
\begin{gathered}
U=\operatorname{diag}(\alpha, \alpha,(\beta) \ldots, \quad(\gamma) \underset{n-5}{n}, \quad, T) \\
\text { times } \\
T=2 \alpha+(n-5) \beta+3 \gamma
\end{gathered}
$$

Note that if the $u_{\text {max }}$ is the nighest value of $U$ in the representation $b$ and if the lowest value is $u_{\max }$ (in a self conjugate representation we have $\sqrt{u_{\max }}=\frac{n-1}{2} \mathrm{~T}$

$$
b=\frac{n-1}{2}
$$

$$
\text { and } 4 \text { max }=2 \text { from inenis meavery }
$$

E.G. (3.6) reduces our freedom of choice to 2 parameters! To construct the states of the representation and denoting by $E$ the set of diagonal operators $I_{3}^{L}, U$ etc. , we have for their eigenvalues

$$
\begin{aligned}
& \Lambda^{0}: e_{\max } \text { (given) } \\
& \Lambda^{1}:\left(i \frac{\text { th }}{\text { state })} e_{\max }-T\left(\theta+E_{i}\right.\right. \\
& \Lambda^{2}:\left(i, j \frac{\text { th }}{\text { state })} e_{\max }-2 T(\varepsilon)+\hat{E}_{i}+E_{j}\right.
\end{aligned}
$$

$E_{i}$ are the sigenvalue $1 E$ in the depiming representeci.

We have sketched here the mechanism for the construction of the representation's vector space. We refer the reader to ref. 6) for proofs and for the actual construction of the representation matrices.

4. Unification: precise generations and the preaiction

$$
\text { of su(3) color. }
$$

It is remarkabie that with such a restricted system, the results should again be so spectacular. Using the choice

$$
\begin{aligned}
& s \ell(n / 1), n-5=k \geq 0 ; \cdot \operatorname{si}(m / 1) \Rightarrow \operatorname{su}(2 / 1) \otimes \operatorname{su}(3)_{c} \eta \\
& b=\frac{n-1}{2} \\
& \mathrm{U}=\operatorname{diag}\left(\frac{-k}{4+k} \cdot \frac{-k}{4+k} \cdot\left(\frac{4}{4+k}\right)_{k \text { times }}\left(\frac{4-2 k}{3(4+k)}\right){ }_{3 \text { times }} \mid \frac{4}{4+k}\right. \\
& I_{3}^{L}=d i a g\left(\left(\frac{1}{3},-\frac{1}{2},(0) k+3\right.\right. \text { times }
\end{aligned}
$$

we get a $2^{n}$-dimensional representation containing precisely $2^{k}$ "generations" of the basic 16 fermion fields. $v$ ma their 16 matter ghosts $\psi^{\mathrm{m}}$. The discrete $\mathrm{s}$ operator in (2.7) is thus embedded in the superalgebra for $n \geq 5$. This set of 32 fields make up the $b=2$ representation of $s l(5 / 1)$, which is itself, reducible 
(though not completely reduciole, a common case in superalgebras). This feature of reducibility and an invariant $2^{n-1}$ subspace occurs for all $s \ell(n / 1)$ if $b$ is an integer. In the case of $s l(5 / 1)$, in approacnes which do not distinguish between fields and ghosts, the irreducible 16-dim. invariant subspace already contains a full set of fields. In either case, the fields sometimes appear through their criarge-conjugates, as in the GeorgiGlashow su(5). However, if we take $s l(6 / 1)$ as the basic physical set, its irreducible 64-dim. $b=5$ representation is chargeconjugation symmetric and contains all 16 fielas, their ghosts and the charge-conjugate states of both. Depending on the treatment, this could be regarded as one, two or four generations. C-symmetry might be important for the removal of Adler-Bell-Jackiw anomalies.

We have introduced color SU(3) through the step sl $(2 / 1) \rightarrow$ $s l(5 / 1)$. Our quantum numbers (4.1) produce an su(5) which does not coincide with the Georgi-Glashow su(5) assignments, since our

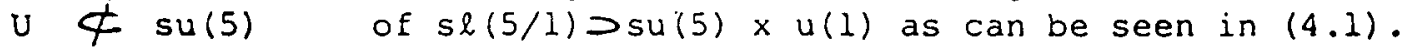
However, it is also possible in $s \ell(5 / 1)$ to identify $U$ with an su(5) quantum number as in the Georgi-Glashow model, as was suggested by A. Salam and J. G. Taylor 16)6).

Beyond this spectacularly unique fit with the generations issue, it has also been snown 18 ) that the supergroup structure requires color to be represented by su(3). Indeed, these fundamental representations impose a unique sub-division in the eigenvalues of $U$ in (4.1), generating the su(3) color group commuting with $s l(2 / 1)$, if we require that the color group su( $r)$. appear in representation $\underline{r}$ for all quarks.

One important result of such a unification is the fact that all the matter fields (and their ghosts, in that interpretation) can be considered as composite. The fundamental constituent is the fractionally-charged $n+1$ dimensional primitive field with $n$ fermions and one ghost. The charges will depend on the final selection for $k$.

In the multigeneration systems beyond $s l(5 / 1)$, the correlation between chirality and the grading vanishes. 
References

1. Y. Ne/eman, Phys. Lett. 813, 190, (1979)

2. D. B. Fairile, Phys. Lett. 228, 97 (1979)

3. L. Corwin, Y. Ne'eman anci S. Sterniderg, Rev. Mod. Phys. 47. 573 (1975)

4. M. Scheunert, W. Nahm ànd $V$. Rittenderg, jour. Matr. Phys. 18,155 (1977)

5. Y. Ne Teman and J. Thierry-Mieg, proceedings of the Saiamanca Internationa: Conference on Differentiai Geometry Methods in Matnematical Physics, to de pubilished by Springer Verlág, Lecture Notes in Matnematics (1979)

6. Y. Ne'eman and S. Sternberg, Proc. Nätl. icad. Sci. USA, in press.

7. J. Thierry-Mieg, These de Doctoral d/Etat (Ovsay, France) (1978)

J. Thierry-Mieg, Jour. Math. Phys. in press (1980)

$\mathrm{J}$. Thierry-Mieg, Nuovo Cim. A. in press (1980)

8. Y. Ne'eman, T. Regge and J. Thierry-Mieg, Proceedings of the $X I X$ International Conference on Hicin Energy Pnysics (Tokyo 1978) eds. S. Homma, M. Kawaglichi and H. Miyazawa Phys. Soc. Jap. Puo. pp. 552-554 (1979)

J. Thierry-Mieg and Y. Ne'eman, Annals of Phys.Vol.123,2 (1979) pp. 247-273.

9. Y. Ne'eman and J. Thierry-Mieg, Proceedings of the VIII international Conference on the Applications of Group Theory to Physics (Kiryat-Anavim 1979) ecis. L. Horwitz and $Y$. Ne'eman, Annals of the Israeli Physical Society (1980)

10.C. Becchi, A. Rouet and R. Stora, Com. Math. Phys. 42, 127 (1975)

11.Y. Ne'eman and I. Thierry-Mieg, Proc. Natl. Acad. Sci. USA 77 (1980)

12.E. J. Squires, Phys. Lett. 82B, 395 (1979)

13.J. G. Taylor, Phys. Lett. $83 \overline{3 B}, 331$ (1979)

14.P. H. Dondi and P. D. Jarvis, Phys. Lett. 84B, 75. (1979)

15.J. G. Taylor, Phys. Lett. 84B, 79 (1979)

16.J. G. Taylor, Fiys. Rev. Lett. 43, 826 (19\%9).

17.M. Gell-Mann and $Y$. Ne'eman, (1976) unpublished, first quoted

in D. 2. Freedman, Phys. Rev. Lett. 38, 105 (1977)

18.Y. Ne'eman and S. Sternberg, to be published. (1980) 